\title{
A Computer Aided System to Improve Dimensional Control of on-site Construction
}

\author{
Rui Wu, Ger Maas \\ Eindhoven University of Technology, Department of Architecture, Building and Planning, P.O. Box \\ 513, 5600 MB Eindhoven, The Netherlands \\ jolleynwu@hotmail.com,g.j.maas@bwk.tue.nl
}

\begin{abstract}
The increased use of prefabricated components, the complexity of new building shapes, and the speeding up of production in construction, demand an efficient and precise dimensional control. Meanwhile Information and Communication Technology (ICT) increasingly supports construction, among others, CAD systems and Total Stations, are being used for the purpose of dimensional control. In order to improve the dimensional control of on-site construction projects, this research tries to capture the knowledge required to design an adequate dimensional control plan and make that knowledge more generally available, and build a connection between CAD systems and Total Stations by applying state-of art ICT, focusing on prefabricated concrete building structural elements. This paper presents an updated information model that defines the various deviations and represents engineering experience knowledge using UML. The model has been implemented in a Dimensional Control System (DCS) and applied in the "La Tour" construction project in Apeldoorn, the Netherlands.
\end{abstract}

KEYWORDS: CAD Systems, Dimensional Control, Prefabricated Concrete, Total Stations, UML

\section{INTRODUCTION}

This paper is based on a research trying to capture the knowledge required to design an adequate dimensional control plan and make that knowledge more generally available, and build a dimensional control system (DCS) facilitating the digital connection between CAD systems and Total Stations. The research has been focused on prefabricated concrete building structural elements.

We have discussed the concepts of dimensional control and an initial model for defining various dimensional deviations in previous publications. Here we will focus on the update of the model and implementation of the DCS as well as its case study in La Tour project.

\section{THE NATURE OF DIMENSIONAL CONTROL PROBLEM}

The essence of dimensional control is to find out the points needed for setting out, positioning and dimension monitoring. In the beginning, one of the research objectives has been to investigate the body of knowledge used by the engineers to design the dimensional control plan. After observations on building sites and interviews with the engineers, contractors and site personnel, it is concluded that there is little explicitly expressed knowledge generally available. That is to say, there are no clear answers to the question of which points to be used for the different aspects of dimensional control including setting out, positioning and dimension monitoring and why. Also, in the Dutch standards for measures and measuring in construction (NEN series 14), the answer cannot be found though it gives some insight to tolerances and deviations.

There is however a lot of factual dimensional control knowledge that resides quite - in the form of rules of thumb - in the heads of experienced planners. On the other hand, there are so many forms of deviations and dimensional differences following different construction methods for various types of projects, that it is not reasonable to expect that the whole body of dimensional control knowledge will ever be fully formalized at all.

The dimensional control problem is basically of a stochastic nature, because every measure originating from construction process has a stochastic deviation and each solution first has to find a way to handle this. Handling the stochastic deviations originating from construction processes like prefabrication, setting out and positioning, is quite problematic for humans but ideally suited for computers. 


\section{POSSIBLE ICT SOLUTIONS FOR DC}

We have studied the state of the art in ICT as seen relevant for the development of a DCS. The main conclusions are:

1) AutoCAD and Visual Basic provide the development environment most suitable for dimensional control (DC). Besides the fact that AutoCAD is market leader in design software of the building industry, it also provides an entrance to the world of Product Data Technology and related next generation tools. With the integration of Visual Basic, a simple but powerful programming language and application builder, it seems possible to build a DCS that can be accessible for the future users to add their own experience to the system. VBA is the programming environment most suited to the job, as it is able to work with a domain specific object model. This object model can be tailored to the needs of DC. Moreover with ADT also a productmodeling interface (IFC) becomes available. Finally there are several ways to import and export VR.

2) Knowledge Technology, especially Rule Based Systems, provides mechanisms suitable for DC to express engineering experience in the form of rules of thumb. Simulation and modeling are suited for prediction and calculation of error propagation of dimensional deviations. Fuzzy logic can be used to add greater flexibility to the expressions if linguistic ambiguity comes into play.

\section{MODELING AND DESIGN OF A DCS}

As mentioned above, there is very little explicitly structured and formalized knowledge available to be put in a knowledge base as a general solution to dimensional control problems. Dimensional uncertainty, dimensional deviation, and the stacking up of deviations springing from several sources form the core of the problem. Following the Monte Carlo simulation technique, we have a method to simulate setting out deviations, positioning deviations and product deviations, and the prediction of the dimensional deviation limits that show the dimensional quality. This method assumes that each individual deviation belongs to a certain range that has a certain percentage of certainty. For example, the deviation can be within the range of , $10 \mathrm{~mm} \pm$ with the certainty of $98 \%$. Each deviation is randomly picked out of certain range and put together. If this is done a large number of times (e.g. 1000 times), the distribution of the total deviation can be predicted. On the other hand, it could still be possible to design a system capable of capturing rules of thumb when they come along.

Another observation has been that the DCS envisioned could also support experienced planners in complicated positioning situations. In fact the use of the DCS in complicated positioning jobs seems to be more attractive than the application of the DCS by inexperienced planners. The idea of this DCS is that a project database holds a detailed description of the facility under construction in a "neutral" (vendor independent) product model format. A product model described in the neutral format describes a facility (building, road, bridge) in the semantics of the construction industry. Product models can be exchanged or accessed by computer networks. The project database is filtered and converted by the DC-Tool to a local database that describes the facility as a collection of physical objects (structural elements, equipment and temporary structures) accompanied by coordinate systems and setting-out points. Next the positioning points are defined on the physical objects, using reference points, and the deviation and tolerance data is analyzed. The DC-Tool feeds the required control data to the Total Station on the construction site.

Based on the ideas mentioned above, we have updated our dimensional control information model by structuring the entities or objects needed for both the simulation of dimensional deviation limits and expression of engineering experience. Also example knowledge rules have been incorporated to the objects. This model is open and more knowledge rules can be added when available. See Figure 1 for the model in UML.

To implement the simulation, we need a random number generator (normal distribution). We have used the following formula to do the job:

$\varepsilon=\sum_{i=1}^{12} R_{i}-6$

where the $R_{i}$ are independent random numbers between 0 and $1(1 \leq \mathrm{i} \leq 12)$ and $\varepsilon$ is the required sample from $\phi(0,1)^{1}$. This approximation can be used to generate variables of standard normal distribution with mean 0 and standard deviation 1

To generate a normally distributed random variable $\mathrm{X}$ with mean and standard deviation, the following formula is used:

$\mathrm{X}=\mu_{\mathrm{x}}+\varepsilon \sigma_{\mathrm{x}}$

$\varphi$ denotes the cumulative distribution function (CDF) of the standard normal variable. 
In the implementation, the above-mentioned method has been used for generating random numbers of deviations that are distributed according to the normal distribution $\mathrm{N}\left(0, \sigma_{\mathrm{x}}\right)$. The range of each deviation variable can be determined according to standards (NEN series 14 ), and $\sigma_{x}$ can be approximated from samples.

When implementing the model into a prototype, we have chosen AutoCAD Architecture Desktop, Access and Excel as the software platform, and VBA as the programming language.

The prototype can generate typical structural assembly or read an IFC format drawing. Figure 2 shows that the tool marks positioning points on an element when the user selects the element. For each possible positioning point of an element, 100 random numbers of normal distribution have been generated (Figure 3 shows the generated numbers in the Access database). The distribution of the predicted deviation limits, which is approximately the normal distribution, can also be shown in Excel. It also gives the $\mathrm{X}, \mathrm{Y}, \mathrm{Z}$ coordinates of the marked positioning points.

\section{CASE STUDY}

The purpose of doing case study is to test the model and prototype, and show the proof of concept. This can be done by using the prototype tool in practice to position several building elements and comparing with the traditional way in the aspect of position accuracy. The data has been collected on the construction site and analyzed in house. Then the conclusion has been made based on the data analysis.

The office-building project "La Tour" concerns an office building with 21 floor levels. Around this building a parking garage of 3 levels will be constructed. The construction of this building will be going on as follows: foundation of prefabricated piles, cellar, in-situ casting concrete core by way of climbing formwork, prefabricated inner wall plates, floor slabs, and further façade elements which will be prepared as much as possible on the construction site.

\subsection{Test}

For this particular project of La Tour, the prototype developed before has been tested and improved. It functions as follows:

The GUI can read AutoCAD drawings of the floor plan and give advices on setting out the core construction horizontally and vertically by asking the user questions. The GUI first defines own coordinate system called RCS. This is because each drawing can have its own coordinate system and everything should be brought back to the same coordinate system. Then the GUI asks the user how many pours of floor there are and the sequence of pouring, and then it gives the number of MOUS points. The location of the MOUS point has relationship with the gridline, usually a fixed offset. Knowing the locations of the points, the system can add them into the floor plan. For vertical setting out, the MOUS points will be transferred upwards. The GUI asks the user to check for obstacles in the space with a range of $150 \mathrm{~mm}$. If obstacles are found, change the offset for everywhere and keep a safe offset; and also be careful with the distortion of signal/light. In the drawing of every floor, MOUS points have the same $\mathrm{X}, \mathrm{Y}$ coordinate.

\subsection{Collecting and Analyzing Data}

The following steps have been carried out on the construction site of La Tour in Apeldoorn to measure and collect data.

1) Select two columns as study objects, one sloped and the other vertical (Figure 4 shows the sloped column on the site).

2) Put the reflecting stickers on two columns. On the sloped column, choose one side surface and find the practical center line of it (Figure 5 shows zooming in of the 3 stickers on the sloped column).

3) Put the reflecting aiming device on the two MOUS points and the reflecting sticker on the line of $1 \mathrm{~m}$ plus.

4) Set up the tripod and the Total Station using Free Station method.

5) Choose "Free Station" in the software of the Total Station.

6) Sight the reflecting stickers on the sloped column and the vertical one, and measuring the $X$, $\mathrm{Y}, \mathrm{Z}$ coordinate of each point.

The final position deviation of an object comes from the deviation of surveying instrument, the deviation of setting out marks including main control points and positioning marks for the object, positioning deviation, and the product deviation of the object. In this case study, the surveying instrument is the Total Station that has a deviation of $2 \mathrm{~mm}$ in distance measurement and $0.0006 \mathrm{~g}$ in angle measurement; the main control points are two MOUS points having a deviation of $2 \mathrm{~mm}$ position; the positioning deviation of objects includes 3 translation and 3 rotation in the 3D space; and the product deviation includes 
deviation in 1D, 2D and 3D. We have analyzed the data by calculating the product deviations and positioning deviations for both the vertical column and sloped column. Figure 6 shows the sloped column with numbered points on one side surface.

\subsection{Conclusions}

The DCS has been applied in the case study by using AutoCAD drawing, Total Station plus storing device and stickers, whereas the traditional technique is paper, theodolite plus notes and measuring tape. Comparing the case method with the traditional technique, you get a list of advantages and disadvantages as follows.

Advantages of the DCS method over traditional technique:

- Speed and accuracy are higher because there are no intermediate points

- Reliability is higher because it reduces the chances of human mistakes by inputting data directly from computer to Total Station instead of paper notes

- Low cost for the overall method

- It can work in 3D where traditional methods cannot

- It works very well with (pre)positioning of free form object in 3D

- Temperature independent

- It can reach NEN very easily

Disadvantages of the DCS method:

- Expensive as a start. Total Station costs NLG 18000

- People with higher quality of knowledge required

Weighing the advantages over disadvantages, the following conclusions on the DCS method can be drawn:

- Higher accuracy

- Less time consuming (around 50\%)

- Highly profitable

- Can be used on all construction works

Therefore the case study shows that the method presented in the model and prototype of the DCS has big benefit for the construction companies if applied appropriately.

\section{CONCLUSIONS}

The conclusions have been summarized as follows:

1) The proposed simulation method and UML information model show a way to handle the dimensional deviations and structure the dimensional control knowledge in a computer interpretable manner. The implementation of a prototype of the DCS provides a digital way to bridge the floor plan design with dimensional control, predict dimensional deviation limits and output the data needed for a Total Station.

2) The case study tests the UML model and prototype of the DCS. The results prove that direct positioning of objects (by putting reflectors on the objects and using a Total Station and by inputting coordinates extracted and calculated from the AutoCAD drawings) provides higher speed, accuracy and reliability. It also shows a way to (pre)position a free form object in 3D where traditional methods cannot. This means that fulfilling the clients' desire to increased freedom in shapes can be supported by the DCS.

3) With the development of IFC standards in the building industry, IFC based building modeling systems will replace current 2D drawing systems, which makes the DC system future proof.

\section{REFERENCES}

Rubinstein, R.Y. (1981). Simulation and the Monte Carlo Method. John Wiley \& Sons, Inc. U.S.A.

Wu, R., P. van Hoof, G. Maas \& F. Tolman (2000). Product Modeling for Dimensional Control in the Building Industry, Computer-Aided Civil and Infrastructure Engineering 15 (2000) 330-341, Blackwell Publishers, U.S.A., 2000.

Wu，R (2002). Computer Aided Dimensional Control in Building Construction. PhD thesis. Eindhoven University of Technology, the Netherlands. 


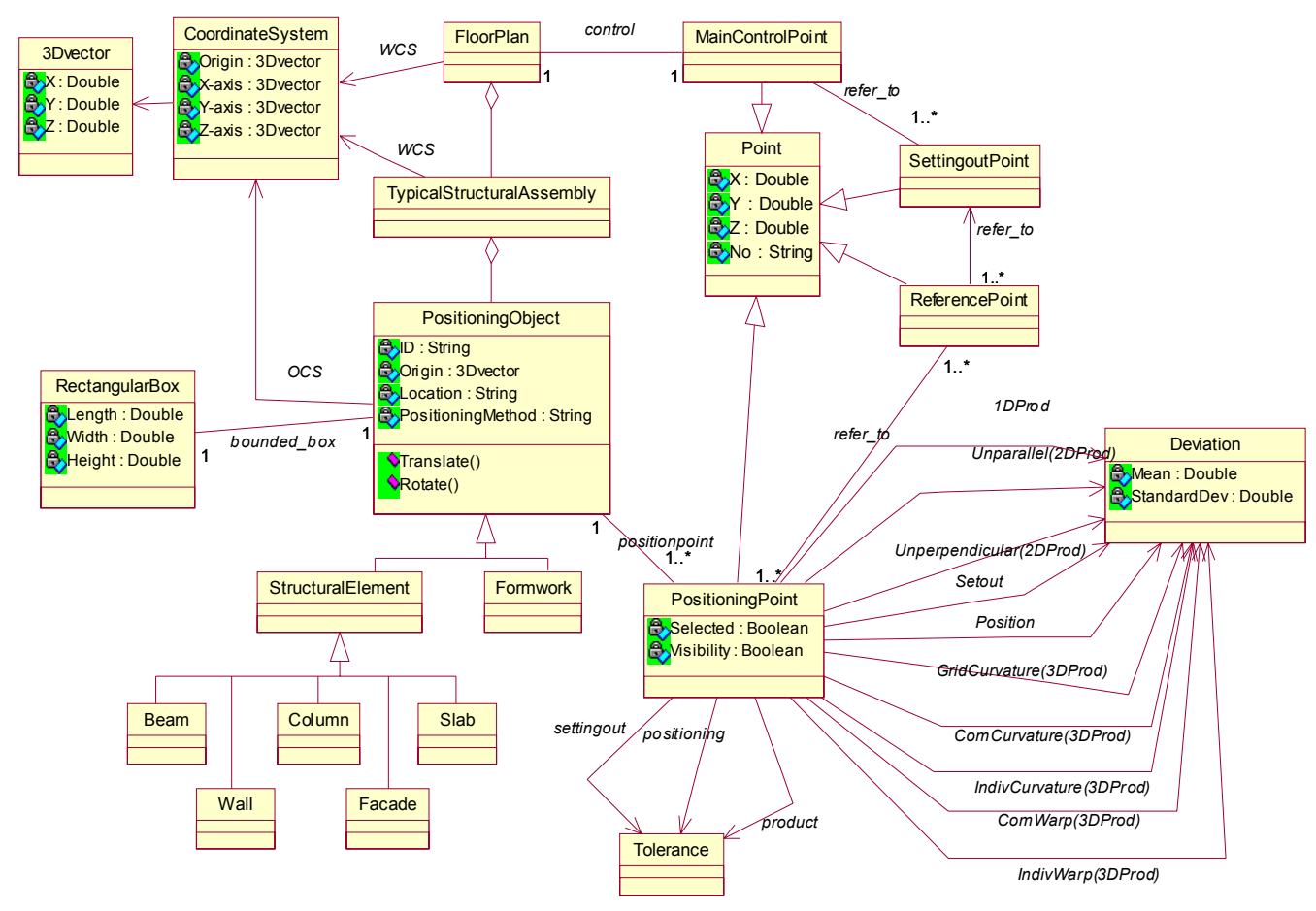

Figure 1. Dimensional Control Information Model in UML

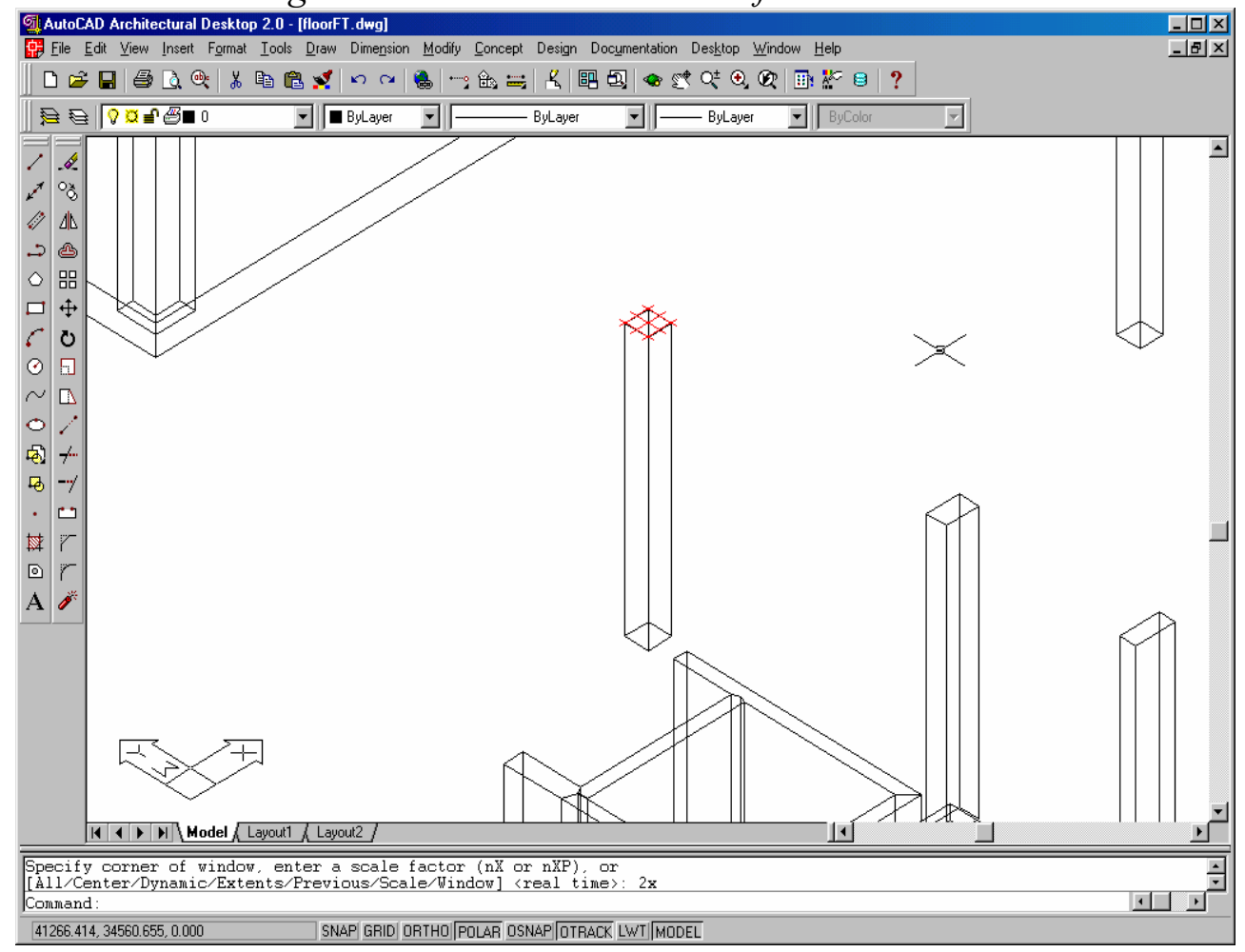

Figure 2. Marking Positioning Points on an Element 


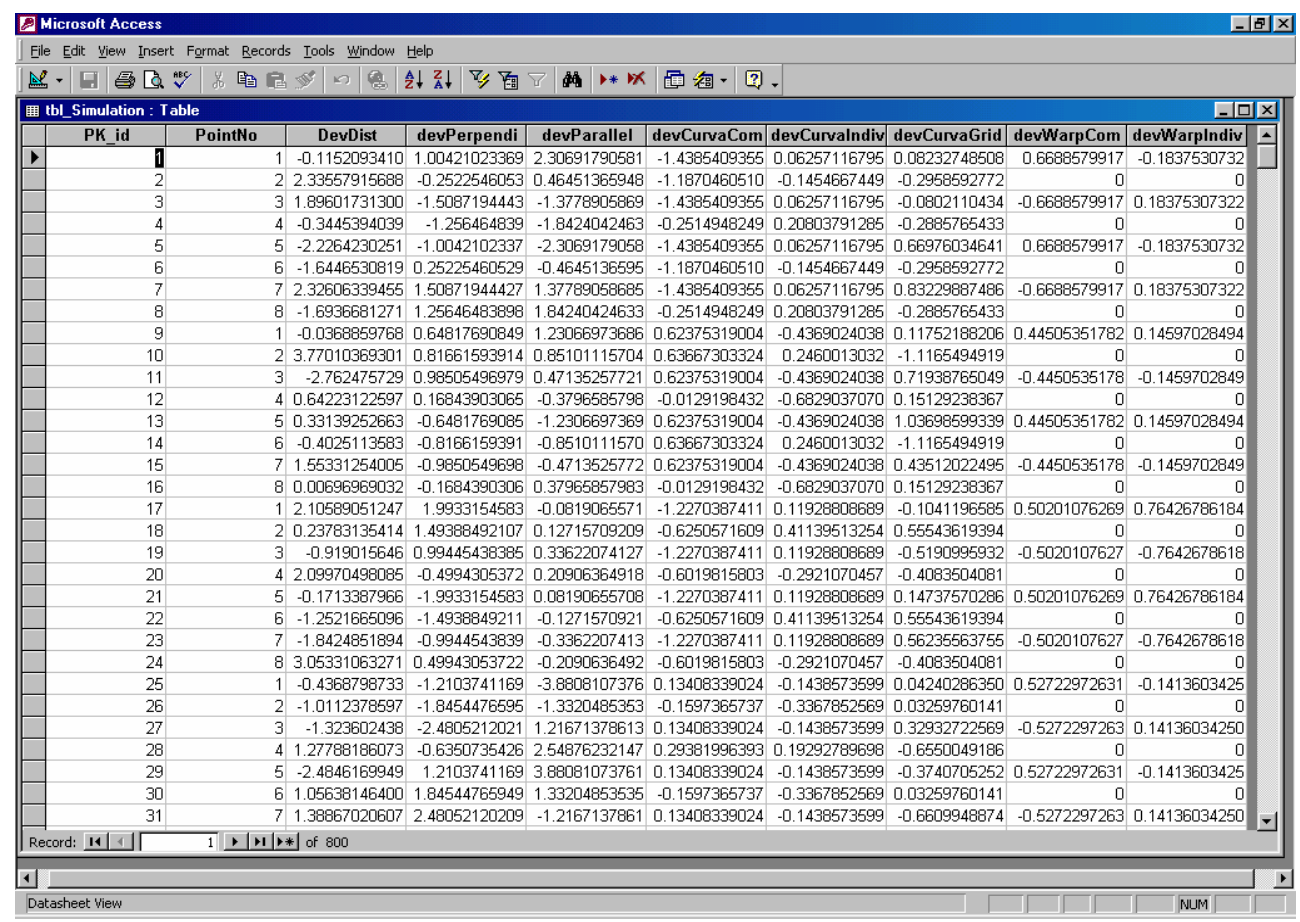

Figure 3. Generated Random Numbers of Normal Distribution in Access Database

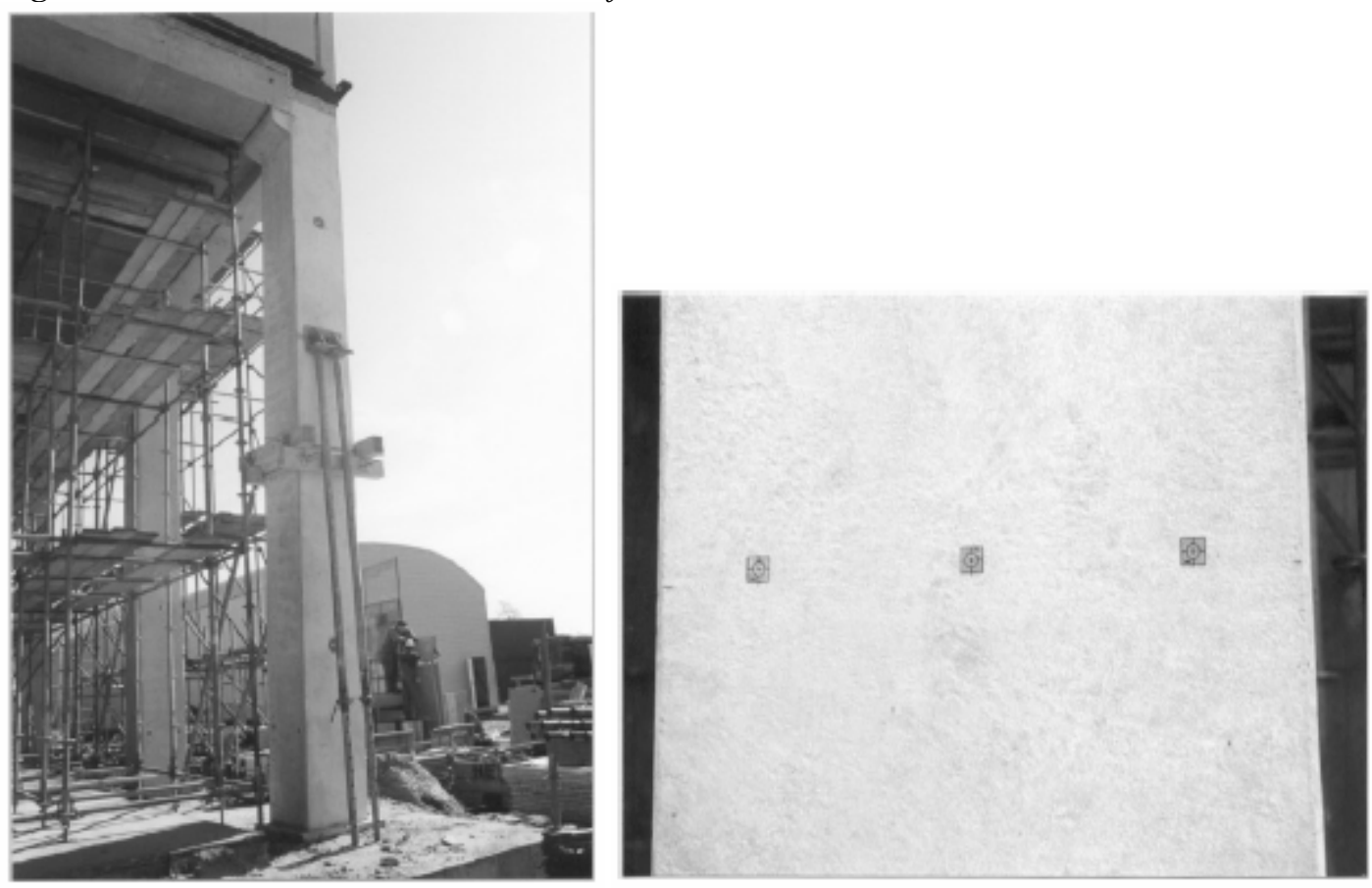

Figure 4. The Sloped Column on the SiteFigure 5 Zooming in of the 3 Stickers on the Sloped Column 


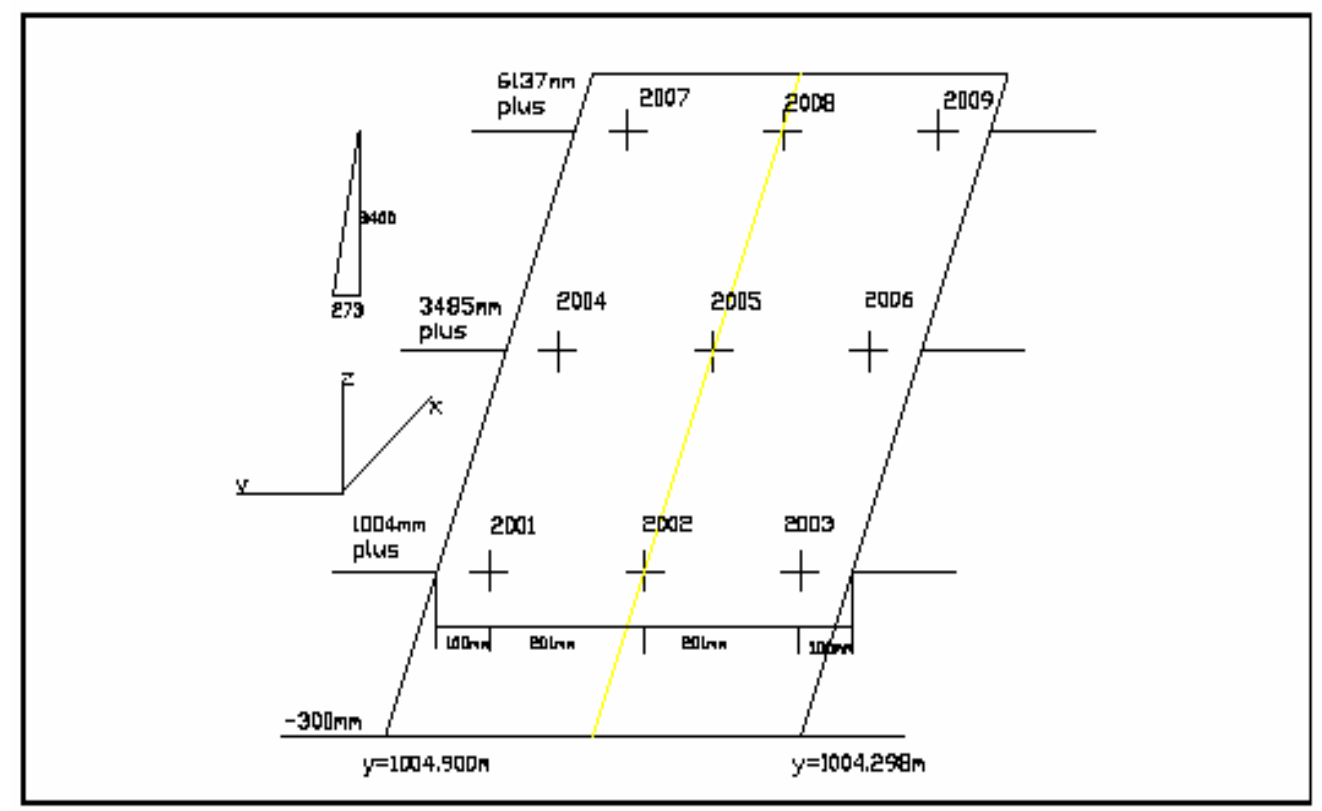

Figure 6. The Sloped Column with Numbered Points on One Side Surface 